\title{
A statistical thermodynamic model of the organizational order of vegetation
}

\author{
Huayong Zhang a, Jianguo $\mathrm{Wu}^{\mathrm{b}, *}$

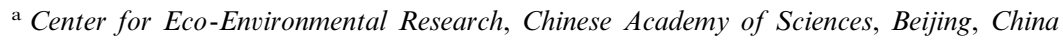 \\ ${ }^{\mathrm{b}}$ Department of Plant Biology, Arizona State University, Tempe, AZ 85287-1601, USA
}

\begin{abstract}
The complex pattern of vegetation is the macroscopic manifestation of biological diversity and the ecological order in space and time. How is this overwhelmingly diverse, yet wonderfully ordered spatial pattern formed, and how does it evolve? To answer these questions, most traditional approaches have been empirical and inductive, based primarily on vegetation classification and gradient analysis. In contrast, here we develop a statistical thermodynamic model of the organizational order of vegetation $(\mathrm{OOV})$ that can be used to derive vegetation patterns at large scales. We define $\mathrm{OOV}$ as a thermodynamic measure of the degree of structural and functional self-organization of natural vegetation in a given environment, which is related to the complexity and stability of ecosystems. The model unites OOV, ecosystem entropy, actual annual evapotranspiration, and mean annual temperature in the same thermodynamic framework. We use the model to calculate OOV values for major world biomes and derive their global pattern. The vegetation gradients predicted by the model contain the major ecoclines empirically identified by Communities and Ecosystems (1975) 385. In addition, the thermodynamic model predicts more gradients of ecosystems at the global scale, which provides theoretical insights into large-scale vegetation pattern analysis. This paper demonstrates that, as we deal with the complexity, diversity, and heterogeneity of ecological systems, statistical and non-equilibrium thermodynamics may serve as both a theoretical framework and a practical modeling approach to integrate patterns and processes across scales. (C) 2002 Elsevier Science B.V. All rights reserved.
\end{abstract}

Keywords: Vegetation pattern and complexity; Organizational order of vegetation; Statistical thermodynamics; Global pattern of vegetation

\section{Introduction}

The study of the diversity and complexity of vegetation pattern has long been an essential and fascinating topic in ecology. On the one hand,

\footnotetext{
* Corresponding author. Tel.: + 1-480-965-1063; fax: + 1480-965-6899.

E-mail address: jingle@asu.edu (J. Wu).
}

vegetation as a crucial part of biodiversity is the result of interactions among numerous biological and physical factors; on the other hand, it provides the fundamental basis for the diverse and complex functionings of ecosystems. While earlier vegetation studies mainly aimed to understand how climatic and soil conditions determine the distribution of vegetation types, recent research has focused on biological diversity, pattern and 
process, patch dynamics, and spatiotemporal vegetation dynamics (Pickett et al., 1987; Wu and Levin, 1994; Crawly, 1997). How do species form diverse vegetation types that exhibit spectacular patterns in space and time over a wide range of scales? This remains a fascinating, yet challenging question in ecology today (May, 1986; Currie, 1991 Levin, 1999). Undoubtedly, the structure, functioning, and dynamics of vegetation are complex, and the complexity of vegetation results from a hierarchy of causes and mechanisms over a range of scales (Pickett et al., 1987; Wu and Loucks, 1995). Simplification is the key to achieving any understanding of complexity (Wu, 1999), and thermodynamics provides a suite of theories and methods to simplify and understand complex phenomena (Nicolis and Prigogine, 1989; Jørgensen et al., 1992; Schneider and Kay, 1994; Jørgensen, 1997; Müller, 1997; Ulanowicz, 1997).

Ecosystems are essentially dissipative systems whose order and metastability in structure and functioning are maintained through continuous negative entropy fluxes from their environment (Nicolis and Prigogine, 1977; Naveh, 1987; Naveh and Lieberman, 1984 O'Neill et al., 1986; Wu, 1991, 1999; Li, 2000). In the past several decades, numerous studies in a variety of fields have indicated that the principles and methods of statistical and non-equilibrium thermodynamics not only are suitable for physical and chemical systems, but also can be used to understand the pattern and process of ecosystems (e.g. Fränzle, 1994; Schneider and Kay, 1994; Jørgensen, 1997; Müller, 1997). In this paper, we develop a mathematical model of the organizational order of natural vegetation using a statistical thermodynamic approach. The organizational order of vegetation $(\mathrm{OOV})$ is a thermodynamic measure of the degree of structural and functional self-organization of natural vegetation in a given environmental setting, which is related to the complexity and stability of ecosystems. First, we develop the thermodynamic equation for OOV and discuss its mathematical properties and ecological implications. Second, based on the OOV equation we derive a series of criteria and conditions for detecting the existence and variation of vegetation pattern. Finally, to enhance our understanding of large-scale vegetation pattern and complexity from a thermodynamic perspective, we compute the OOV values for 22 major world biomes that were identified by Whittaker (1975), and deduce the global pattern of vegetation distribution in terms of OOV gradients.

\section{A statistical thermodynamic model of the organizational order of vegetation}

Statistical thermodynamics relates average molecular properties to macroscopic thermodynamic properties such as temperature, pressure, work, heat, and entropy, and its goal is to understand and interpret the macroscopic properties of systems in terms of the properties of their constituent particles (Hill, 1960; Callen, 1985). Thus, statistical thermodynamics couples attributes of systems at different scales. To develop a statistical thermodynamic model of vegetation pattern, we start with the well-known Boltzmann equation, which describes the relationship between the entropy and order of an isolated or closed system i.e.

$S=k \ln W$

where $S$ is the entropy of a system, $k$ is Boltzmann's constant $\left(k=1.3805 \times 10^{-23} \mathrm{~J} \mathrm{~K}^{-1}\right)$, and $W$ is the 'number of complexions' of the system or the probability of a collection of system states (Atmar, 2001). In a hierarchical system (e.g. cells - tissues - organs - individuals - populations -communities), $W$ generally decreases with increasing organizational levels; that is the total number of patterns that can possibly be formed by the components declines at each higher level. In other words, the organizational order of the system increases and more 'organized' spatiotemporal patterns, as opposed to random or disorderly variations, emerge at higher levels.

According to Boltzmann's order principle, an increase in system's entropy necessarily and positively corresponds to a decrease in system's organization, and $W$ in Eq. (1) can be understood as a micro-scale measure of the macro-scale disorder of the system. Furthermore, a thermodynamic 
equilibrium is reached when entropy increases to its maximum (and so does the number of complexions) in isolated systems, whereas in closed systems equilibrium structures (e.g. crystal structures) can be formed when the free energy (free energy $=$ total energy - temperature $\times$ system entropy) reaches a minimum value. Boltzmann's order principle is not applicable to dissipative structures such as ecosystems that are open and far from the thermodynamic equilibrium. However, when the concept of negative entropy is introduced, as in Schrödinger (1944) and Nicolis and Prigogine (1977), Boltzmann equation can be used to describe open systems that have constant energy and material exchanges with environment. In this case, instead of using $S$ to measure the degree of system disorganization, $-S$ can be related to the degree of system organization.

Let us assume that ecosystems can be studied as thermodynamic systems composed of a large number of biological particles (organisms) and that the large-scale patterns of natural vegetation are manifestations of the steady states these biological particles have reached in relation to their environment. At the first glance, these assumptions may seem overly simplified and hard to be justified ecologically. However, similar assumptions have been successfully used in other studies. For example, Durrett and Levin (1994a,b) modeled ecosystems as particle systems to understand how large-scale patterns emerge as the results of localscale interactions (also see Levin (1999)), while Volterra (1926) treated predator and its prey populations as particles to explore the behavior of the ecological community. Based on the above statistical thermodynamic assumptions of ecosystems, we can derive the equation for the OOV by modifying the Boltzmann equation, which takes the following form:

$-S=k \ln (1 / W)$

where $-S$ is the negative entropy required for the development and maintenance of vegetation $\left(\mathrm{J} \mathrm{K}^{-1}\right), k$ is the Boltzmann's constant, and $\ln (1 /$ $W$ ) is defined as the OOV, which may be perceived as a macroscopic, thermodynamic representation of the steady state reached between vegetation and its environment over large spatial and temporal scales.
Eq. (2) indicates that the order of vegetation can be measured by the total sum of entropy fluxes generated by biotic and abiotic components of the ecosystem. However, to apply this equation, we must be able to quantify $-S$. Empirical ecological data have shown that the large-scale patterns of vegetation distribution, primary productivity, and species richness tend to be positively correlated to the actual evapotranspiration (AET) of ecosystems (e.g. Holdridge, 1947; Rosenzweig, 1968; Whittaker, 1975; Currie and Paquin, 1987; Currie, 1991). Thus, we can assume that, at large spatial scales, the negative entropy produced through the actual AET of ecosystems constitutes the major portion of $-S$ in Eq. (2). From a thermodynamic perspective, the negative entropy generated by AET is dependent on the phase transition from liquid water to water vapor in ecosystems. Therefore, to approximate ecosystem's negative entropy using AET, we have:

$-S=\lambda \gamma A \mathrm{AET} / T$

where $\lambda$ is the latent heat of vaporization for water $\left(2.4987 \times 10^{3} \mathrm{~J} \mathrm{~g}^{-1}\right), \gamma$ is the specific gravity of water $\left(1.0 \mathrm{~g} \mathrm{~cm}^{-3}\right), A$ is the area of the ecosystem $\left(\mathrm{cm}^{-2}\right)$, AET is the actual evapotranspiration (cm), and $T$ is the thermodynamic or absolute temperature in Kelvin (K).

From Eq. (2) and Eq. (3), we obtain a general equation of the OOV:

$\mathrm{OOV}=\ln (1 / W)=h A \mathrm{AET} / T$

where OOV is the organizational order of vegetation, and $h=\lambda \gamma / k=1.8099 \times 10^{26}\left(\mathrm{~K} \mathrm{~cm}^{-2}\right)$.

From Eq. (4), we can make the following inferences: (1) In the three-dimensional thermodynamic state space formed by $A$, AET and $T$, OOV is a holistic measure of the large-scale organization of ecosystems, describing the statistical thermodynamic steady state that is created and maintained by the interactions among a large number of biological particles and their environmental constraints on multiple scales. (2) In a non-equilibrium thermodynamic framework, the order of vegetation is positively related to the area and the actual AET, but negatively related to the thermodynamic temperature, of ecosystems. (3) The validity of Eq. (4) is supported by dimen- 
sional analysis and empirical data obtained at large spatial scales (e.g. regional and global), and thus it should be applicable to the study of the order of vegetation and its spatial patterns at these scales. (4) The values of OOV, typically large as with many thermodynamic variables, each corresponding to a point in the thermodynamic state space, change continuously and nonlinearly.

Let's suppose that $A$ is a constant: $A_{0}=0.1$ ha (i.e. $10^{7} \mathrm{~cm}^{2}$ ), which is the sample area frequently used by Whittaker (1975) in his gradient analysis of vegetation. Imagine a reference point that corresponds to this area in the two-dimensional thermodynamic state space composed of AET and $T$. The value of OOV at this reference point $\left(\mathrm{OOV}_{0}\right)$ can be expressed as: $\mathrm{OOV}_{0}=h A \mathrm{AET} / T=h^{\prime} b$, where $h^{\prime}=1.8099 \times 10^{33} \mathrm{~K} \mathrm{~cm}^{-1}$ and $b=\mathrm{AET}_{0} /$ $T_{0} ; b$ may be interpreted as a measure of the efficiency of negentropy production by evapotranspiration at the reference point. If OOV represents the value of the OOVat any point in the AET $-T$ thermodynamic state space, then we obtain the following equation that describes the deviation between this arbitrary point and the reference point:

$\mathrm{OOV}^{\prime}=\mathrm{OOV}-\mathrm{OOV}_{0}=h^{\prime}(\mathrm{AET} / T-b)$

Here, we term $\mathrm{OOV}^{\prime}$ the gradient of the OOV. The importance and utility of Eq. (5) will be explored in the following sections.

\section{Thermodynamic conditions for vegetation development and OOV gradients}

In Section 2, we derived the equations for the OOV and its gradient (OOV') in terms of $A$, AET and thermodynamic temperature $(T)$ of ecosystems. In the following, we will analyze these equations and deduce the general thermodynamic conditions for the existence of vegetation and its gradients.

From Eq. (4) and Eq. (5), it is evident that, when the thermodynamic temperature of ecosystems $(T)$ is zero, the term, AET $/ T$, is meaningless, and so is OOV. Whenever there exists vegetation, $A$ and AET must be larger than zero, and thus
OOV $>0$. At large scales, different vegetation types should have their characteristic values of OOV as the result of plant-environment interactions and thermodynamics constraints. From Eq. (5), we can make the following inferences:

(1) When the value of OOV in the AET-T two-dimensional state space is equal to that of the reference point $\left(\mathrm{OOV}_{0}\right)$, there does not exist a gradient of the order of vegetation (i.e. OOV' = 0 ). This is a thermodynamic description or quantification of spatial (and temporal) homogeneity of vegetation, or the stability of vegetation order. While the order of vegetation is determined primarily by the ratio of $\mathrm{AET} / T$, a relationship of equivalence exists between AET and $T$ : a change of $1 \mathrm{~cm}$ in AET is equivalent to a change of $b$ in $T$. Thus, the order of vegetation may still be the same for two ecosystems which have different AET and $T$, but the same ratio of AET to $T$.

(2) If $\mathrm{OOV}^{\prime}<0$ i.e. $h^{\prime}(\mathrm{AET} / T-b)<0$, then AET $/ T<b$. This indicates a decreasing gradient of the order of vegetation moving away from the reference point in the AET- $T$ state space. There are five possibilities for the formation of this gradient: (i) AET decreases, $T$ increases, thus OOV'<0; (ii) AET decreases, $T$ remains unchanged, thus OOV' $<0$; (iii) AET and $T$ both decrease, but the former decreases faster than the latter, again resulting in $\mathrm{OOV}^{\prime}<0$; (iv) AET remains unchanged, $T$ increases, thus $\mathrm{OOV}^{\prime}<0$; and (v) both AET and $T$ increase, but the latter increases faster than the former, thus leading to $\mathrm{OOV}^{\prime}<0$.

(3) If $\mathrm{OOV}^{\prime}>0$ i.e. $h^{\prime}(\mathrm{AET} / T-b)>0$, then AET $/ T>b$. This indicates an increasing gradient of the order of vegetation moving away from the reference point in the AET- $T$ state space. There are also five possibilities in this case: (i) AET increases, $T$ decreases, thus OOV' $>0$; (ii) AET increases, $T$ remains unchanged, thus OOV' $>0$; (iii) AET and $T$ both increase, but the former increases faster than the latter, thus OOV' $>0$; (iv) AET remains unchanged, $T$ decreases, thus OOV'>0; and (v) both AET and $T$ decrease, but the latter decreases faster than the former, thus $\mathrm{OOV}^{\prime}>0$.

The above inferences provide a thermodynamic basis for describing large-scale vegetation patterns 
and detecting vegetation gradients in terms of the OOV. By calculating the surface (or time series) of $\mathrm{OOV}$, we can analyze the variation of vegetation in space (or time). In the following section, we will demonstrate how to use the thermodynamic models (Eq. (4) and Eq. (5)) and the associated inferences to deduce the organizational order and patterns of global vegetation.

\section{Global pattern of the organizational order of vegetation}

\subsection{Organizational order of global vegetation types}

Taking an inductive and empirical approach, Whittaker (1975) elegantly depicted the pattern of 22 major world biomes in a two-dimensional coordinate system composed of mean annual precipitation and mean annual temperature, and identified four major ecoclines i.e. gradients of communities-and-environments or gradients of ecosystems on a world-wide scale. Undoubtedly, Whittaker's seminal work was monumental in vegetation science, and has had enormous impacts on generations of ecologists until today. However, Whittaker's model is purely empirical. His coordinate system is of the Newtonian space in which variables are independent, and is only meaningful for delineating the boundaries between vegetation classes. As a consequence, vegetation types in this coordinate system are not directly comparable. Furthermore, although the four global vegetation gradients observed by Whittaker are exemplary and ecologically profound, with such empirically based, inductive approaches it is difficult, if ever possible, to answer the question: Are there other possible ecoclines?

Here, we attempt to address these problems using the thermodynamic models developed in the previous sections. First, let's consider how to obtain the values of AET in the thermodynamic models at the global scale. From empirical studies of terrestrial systems, we know that the mean annual AET of ecosystems is equal to the mean annual precipitation (PR) minus the sum of the mean annual surface runoff (SR) and the mean annual penetration (AP) into soil and ground water (e.g. Seller, 1965). Because we do not have data on SR and AP, in the following calculations, we assume the relationship: AET $=\eta \mathrm{PR}$, where $\eta$ represents the percentage of annual precipitation converted into AET (here we let $\eta=1.0,0.8$, and 0.5 , respectively). By so doing, we can use the thermodynamic models to calculate the OOV for major world biomes and derive their global pattern based on the empirical data provided in Whittaker (1975). The results are depicted in Figs. 1 and 2 and Fig. 3, and the values of OOV and OOV' for the 22 biomes corresponding to the three $\eta$ values are listed in Table 1 and Table 2.

Our results show that, at the global scale, the highest value of the OOV occurs in the tropical rainforest (Fig. 1 and Table 1). If annual precipitation is all used for evapotranspiration (i.e. $\eta=$ $1.0)$, the highest value of OOV is $2.7105 \times 10^{33}$. The highest value declines to $2.1684 \times 10^{33}$ when $\eta=0.8$, and to $1.3553 \times 10^{33}$ when $\eta=0.5$. From empirical studies (e.g. Whittaker, 1975 Barbour and Billings, 2000), a 100\% conversion from precipitation to transpiration is impossible in the tropical rainforest, but a $50 \%$ or higher conversion rate is possible. Therefore, we infer that the actual highest value of OOV for global vegetation lies between $1.3553 \times 10^{33}$ and $2.7105 \times 10^{33}$. The lowest value of OOV, on the other hand, occurs in the deserts. Whittaker (1975) indicated that the subtropical desert did not have any vegetation existing when annual precipitation was below 2 $\mathrm{cm}$. We chose a point within the subtropical desert region that corresponds to the PR of mean annual precipitation of $2 \mathrm{~cm}$ and the mean annual temperature of $293.15 \mathrm{~K}$. The $\mathrm{OOV}$ value is $0.0123 \times 10^{33}$ when $\eta=1.0$, and drops to $0.0099 \times 10^{33}$ for $\eta=0.8$ and to $0.0062 \times 10^{33}$ for $\eta=0.5$.

Whittaker (1975) described four major ecoclines. These ecoclines can be identified and quantified with OOV values (Fig. 1; when $\eta=$ 0.8): (1) Temperate rainforest $\rightarrow$ temperate deciduous forest $\rightarrow$ temperate evergreen forest $\rightarrow$ temperate woodland $\rightarrow$ temperate scrubland $\rightarrow$ temperate semidesert $\rightarrow$ true desert; its corresponding OOV gradient is: $1.2075 \times 10^{33} \rightarrow$ 
$0.7427 \times 10^{33} \rightarrow 0.7427 \times 10^{33} \rightarrow 0.3806 \times 10^{33}$ $\rightarrow 0.2238 \times 10^{33} \rightarrow 0.1311 \times 10^{33} \rightarrow 0.0648 \times 10^{33}$. This ecocline corresponds to an increasing gradient in aridity. (2) Tropical rainforest $\rightarrow$ tropical seasonal forest $\rightarrow$ tropical broadleaf woodland $\rightarrow$ tropical scrub $\rightarrow$ warm semidesert $\rightarrow$ true desert; the corresponding OOV gradient is: $1.5914 \times 10^{33}$ $\rightarrow 0.9928 \times 10^{33} \rightarrow 0.5415 \times 10^{33} \rightarrow 0.3217$ $\times 10^{33} \rightarrow 0.1682 \times 10^{33} \rightarrow 0.0648 \times 10^{33}$. This ecocline also corresponds to an increasing gradient in aridity. (3) Tropical rainforest $\rightarrow$ temperate rainforest $\rightarrow$ elfinwood $\rightarrow$ alpine scrubland $\rightarrow$ alpine

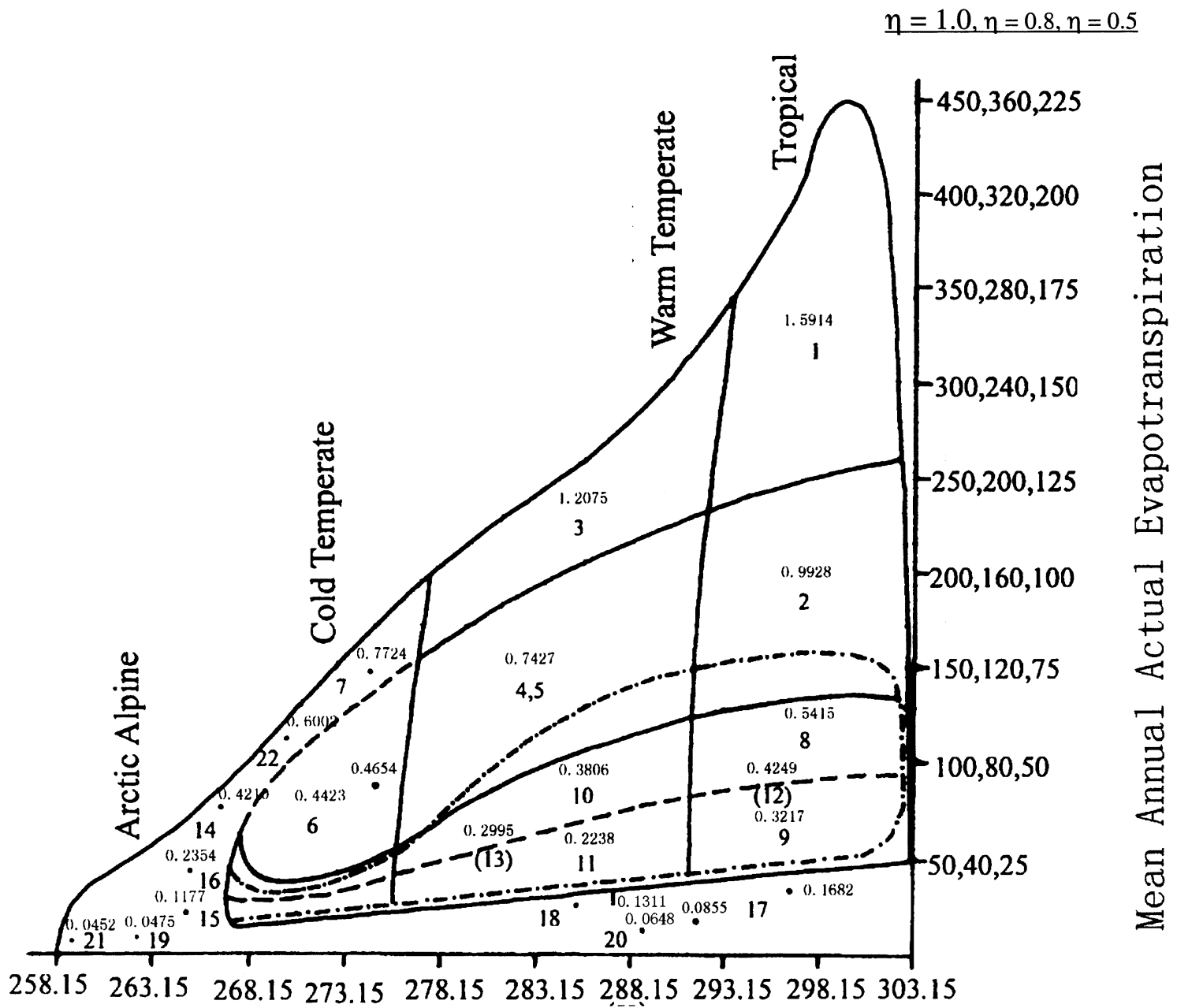

\section{Mean Annual Thermodynamical Temperature}

Fig. 1. The OOV of the major world biomes as classified by Whittaker (1975): 1-tropical rainforest, 2-tropical seasonal forest, 3-temperate rainforest, 4-temperate deciduous forest, 5-temperate evergreen forest, 6-taiga, 7-elfinwood, 8-tropical broadleaf woodland, 9-thorn scrub, 10-temperate woodland, 11-temperate shrubland, 12-savanna, 13-grassland, 14alpine shrubland, 15-alpine grassland, 16-tundra, 17-warm semidesert, 18-cool semidesert, 19-arctic alpine semidesert, 20 - true desert, 21 - arctic alpine desert), 22-cool temperate bog. The larger numbers denote different biomes, whereas the numbers with decimal points are the average values of OOV for the 22 biomes when $\eta=0.8$. For those biomes that do not have closed boundaries, the value of the marked point for each type is reported. The actual OOV values are those in the figure times $10^{33}$. 


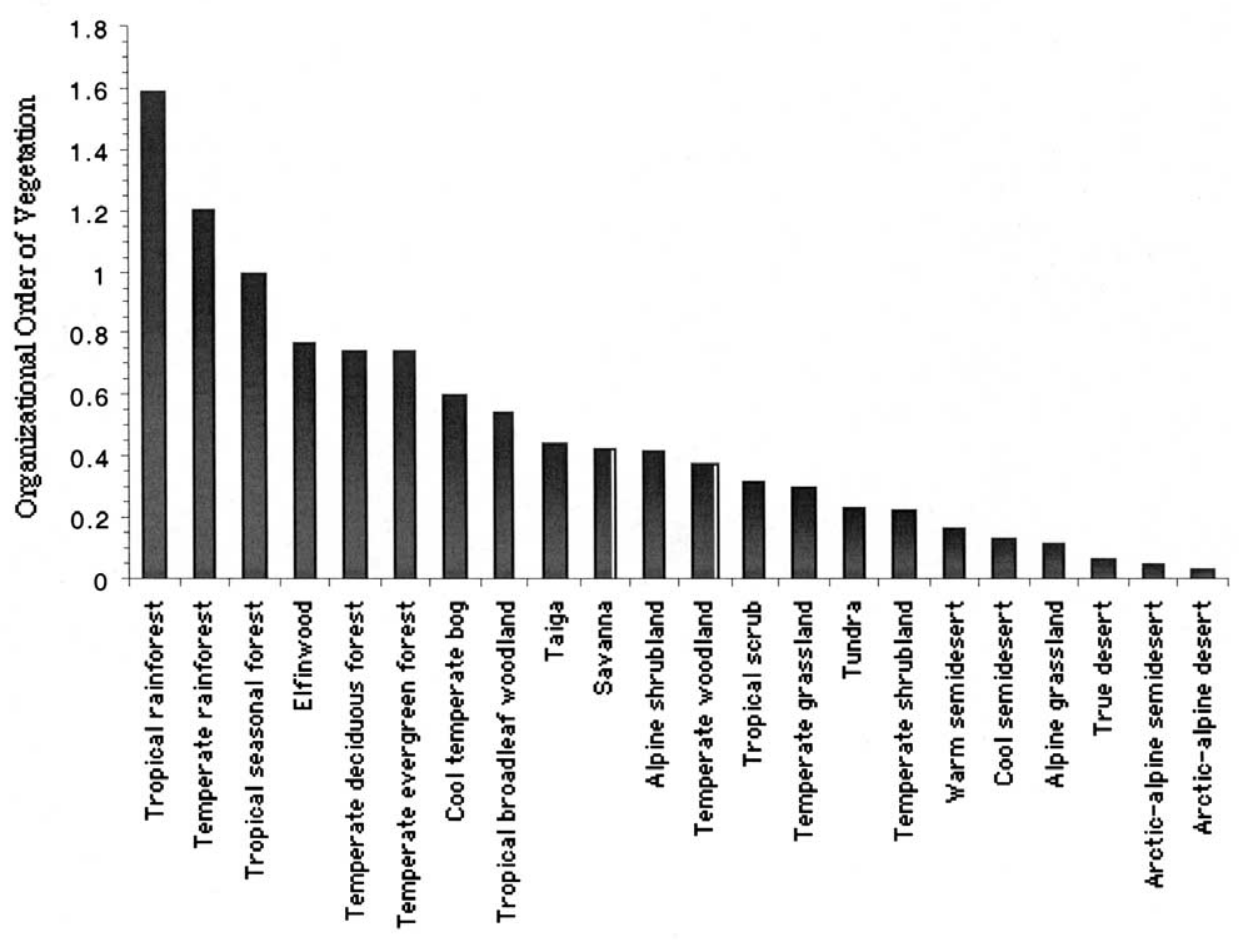

World Biomes

Fig. 2. The OOV values of the major world biomes arranged in a descending order $(\eta=0.8)$.

grassland; the corresponding OOV gradient is: $1.5914 \times 10^{33} \rightarrow 1.2075 \times 10^{33} \rightarrow 0.7724 \times 10^{33} \rightarrow$ $0.4210 \times 10^{33} \rightarrow 0.1177 \times 10^{33}$. This ecocline corresponds to an elevation gradient. (4) Tropical seasonal forest $\rightarrow$ temperate deciduous forest $\rightarrow$ temperate evergreen forest $\rightarrow$ taiga $\rightarrow$ tundra; the corresponding OOV gradient is: $0.9928 \times 10^{33} \rightarrow 0.7427$ $\times 10^{33} \rightarrow 0.7427 \times 10^{33} \rightarrow 0.4423 \times 10^{33}$ $\rightarrow 0.2354 \times 10^{33}$. This ecocline corresponds to a decreasing temperature gradient. In addition, a coniferous forest $\rightarrow$ warm desert ecocline $\left(0.4654 \times 10^{33} \rightarrow 0.0855 \times 10^{33}\right)$ corresponds to the vertical vegetation gradient of the Santa Catalina Mountains of southeastern Arizona, the United States (Whittaker, 1975).

\subsection{Global vegetation gradients derived from the OOV model}

If we choose a point, $\mathrm{O}$, in Fig. 1 as the reference point, then we can construct global transects that describe vegetation changes using the thermodynamic model derived in this paper. To follow the way Whittaker (1975) constructed the figure of world biome distribution, here we choose the coordinates of point $\mathrm{O}$ as $T_{0}=297.28 \mathrm{~K}$ and $\mathrm{AET}_{0}=355.26 \mathrm{~cm}$, and then derive the global pattern of vegetation distribution in terms of OOV. The results are shown in Fig. 3 and Table 2.

Based on our calculations, 31 distinctive global transects, originating from the reference point $(\mathrm{O})$, can be drawn, which belong to 11 types that in turn form three groups (Fig. 3 and Table 2). Group 1 contains only 1 type with 1 transect $\left(\mathrm{L}_{0}\right)$. This transect has an OOV' value of zero, indicating no change in OOV. Group 2 has 5 types and 25 transects, which depicts the trend of vegetation changing from higher to lower order of organization. Group 3 includes 5 types and 5 transects, 
showing the opposite trend: vegetation changes from lower to higher order of organization. Changes in OOV are correlated with changes within and between vegetation types. In general, the gradient of OOV is relatively small within the same biome or between biomes that are similar in vegetation physiognomy. The maximum value of the gradient of OOV within tropical rainforest is $0.4326 \times 10^{33}$ for $\eta=1.0 ; 0.3461 \times$
$10^{33}$ for $\eta=0.8$; and $0.2163 \times 10^{33}$ for $\eta=0.5$. However, the magnitude of OOV' increases between tropical rainforest and other biomes. For example, the value of the OOV gradient reaches its maximum as the vegetation type changes from tropical rainforest to desert: $\mathrm{OOV}^{\prime}=-$ $2.1506 \times 10^{33}$ for $\eta=1.0 ; \quad \mathrm{OOV}^{\prime}=-1.7205 \times$ $10^{33}$ for $\eta=0.8$; and OOV' $=-1.0753 \times 10^{33}$ for $\eta=0.5$.

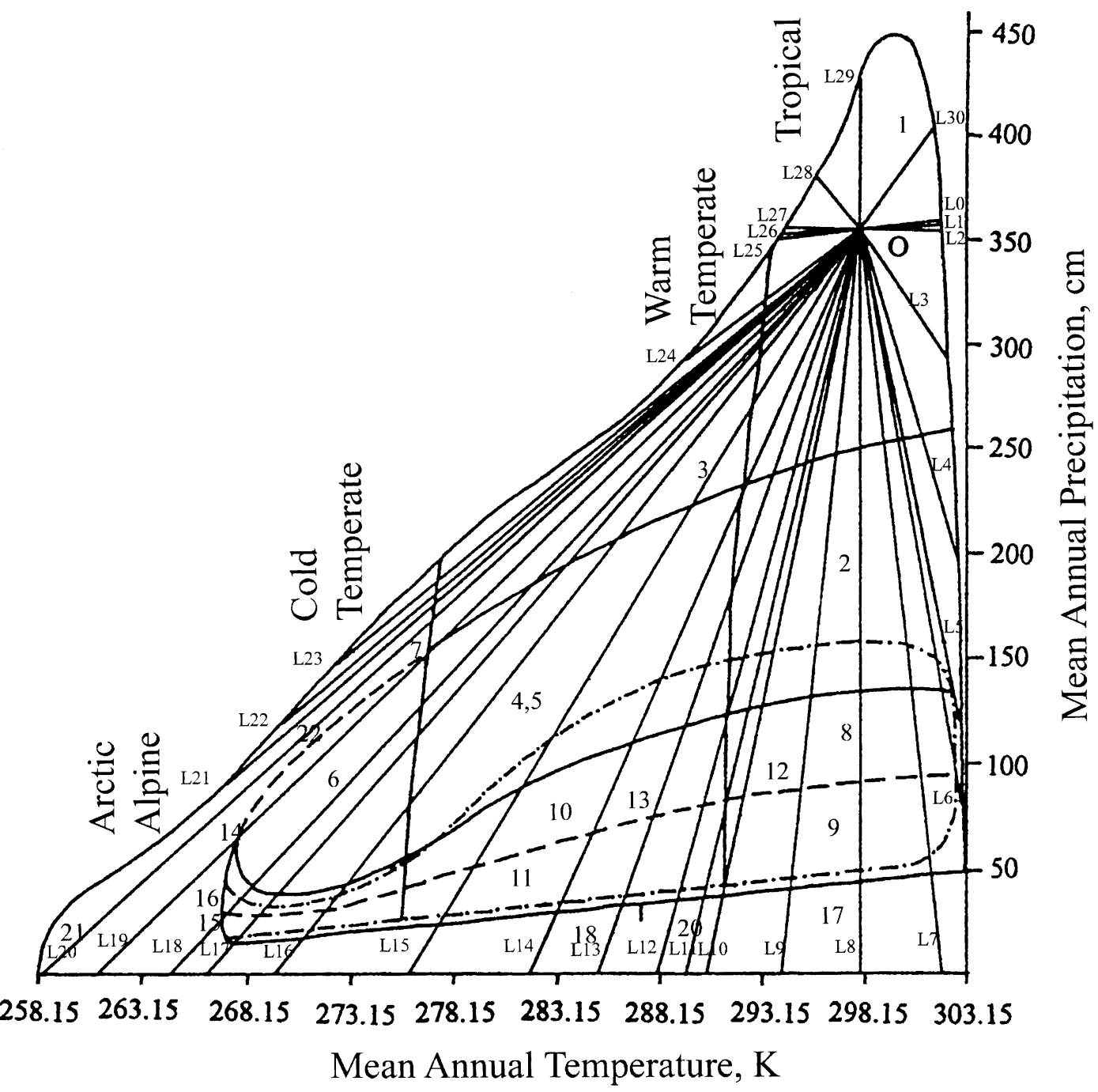

Fig. 3. The gradients of the OOV at the global scale. $\mathrm{L}_{0}, \ldots, \mathrm{L}_{30}$ denote 31 transects (or gradients) that cut across different world biomes. 
Table 1

The values of the OOV for 22 world biomes $\left(\times 10^{33}\right)$

\begin{tabular}{|c|c|c|c|c|c|c|c|c|c|c|c|c|}
\hline \multirow[t]{2}{*}{ Biome type } & \multicolumn{4}{|c|}{ OOV when $\eta=1.0$} & \multicolumn{4}{|c|}{ OOV when $\eta=0.8$} & \multicolumn{4}{|c|}{ OOV when $\eta=0.5$} \\
\hline & Avg & Min & Max & $\mathrm{SD}$ & Avg & Min & Max & SD & Avg & Min & Max & SD \\
\hline 1 & 1.4571 & 1.9892 & 2.7105 & 0.3112 & 1.5914 & 1.1657 & 2.1684 & 0.2489 & 0.9946 & 0.7286 & 1.3553 & 0.1556 \\
\hline 2 & 1.1535 & 0.7806 & 1.5514 & 0.2036 & 0.9928 & 0.2645 & 1.2411 & 0.1628 & 0.5767 & 0.3903 & 0.7757 & 0.1018 \\
\hline 3 & 1.5094 & 1.0187 & 2.0930 & 0.2231 & 1.2075 & 0.8150 & 1.6744 & 0.1785 & 0.7547 & 0.5093 & 1.0465 & 0.1116 \\
\hline 4 & 0.9283 & 0.3801 & 1.4311 & 0.2233 & 0.7427 & 0.3041 & 1.1449 & 0.1787 & 0.4642 & 0.1900 & 0.7156 & 0.1117 \\
\hline 5 & 0.9283 & 0.3801 & 1.4311 & 0.2233 & 0.7427 & 0.3041 & 1.1449 & 0.1787 & 0.4642 & 0.1900 & 0.7156 & 0.1117 \\
\hline 6 & 0.5529 & 0.2536 & 0.9634 & 0.1715 & 0.4423 & 0.2029 & 0.7707 & 0.1372 & 0.2764 & 0.1268 & 0.4817 & 0.0858 \\
\hline 7 & 0.9655 & - & - & - & 0.7724 & - & - & - & 0.4828 & - & - & - \\
\hline 8 & 0.6769 & 0.5266 & 0.8168 & 0.0757 & 0.5415 & 0.4213 & 0.6534 & 0.0606 & 0.3384 & 0.2633 & 0.4084 & 0.0378 \\
\hline 9 & 0.4021 & 0.2354 & 0.5608 & 0.0849 & 0.3217 & 0.1883 & 0.4486 & 0.0679 & 0.2010 & 0.1177 & 0.2804 & 0.0425 \\
\hline 10 & 0.4758 & 0.1890 & 0.7646 & 0.1448 & 0.3806 & 0.1512 & 0.6117 & 0.1158 & 0.2379 & 0.0945 & 0.3823 & 0.0724 \\
\hline 11 & 0.2797 & 0.1012 & 0.5097 & 0.0932 & 0.2238 & 0.0810 & 0.4078 & 0.0746 & 0.1399 & 0.0506 & 0.2549 & 0.0466 \\
\hline 12 & 0.5312 & 0.2354 & 0.8168 & 0.1592 & 0.4249 & 0.1883 & 0.6534 & 0.1273 & 0.2656 & 0.1177 & 0.4084 & 0.0796 \\
\hline 13 & 0.3744 & 0.1012 & 0.7646 & 0.1556 & 0.2995 & 0.0810 & 0.6117 & 0.1245 & 0.1872 & 0.0506 & 0.3823 & 0.0778 \\
\hline 14 & 0.5262 & - & - & - & 0.4210 & - & - & - & 0.2631 & - & - & - \\
\hline 15 & 0.1471 & - & - & - & 0.1177 & - & - & - & 0.0736 & - & - & - \\
\hline 16 & 0.2942 & - & - & - & 0.2354 & - & - & - & 0.1471 & - & - & - \\
\hline 17 & 0.2102 & - & - & - & 0.1682 & - & - & - & 0.1051 & - & - & - \\
\hline 18 & 0.1639 & - & - & - & 0.1311 & - & - & - & 0.0819 & - & - & - \\
\hline 19 & 0.0594 & - & - & - & 0.0475 & - & - & - & 0.0297 & - & - & - \\
\hline 20 & 0.0810 & - & - & - & 0.0648 & - & - & - & 0.0405 & - & - & - \\
\hline 21 & 0.0452 & - & - & - & 0.0362 & - & - & - & 0.0226 & - & - & - \\
\hline 22 & 0.7502 & - & - & - & 0.6002 & - & - & - & 0.3751 & - & - & - \\
\hline
\end{tabular}

The classification of world biomes is based on Whittaker (1975). The names of these biomes are given in Fig. 1. For those biomes with closed boundaries, the average (Avg), minimum (Min), and maximum (Max) values as well as standard deviation (SD) of OOV are given; for those without closed boundaries, only the values of OOV at the representative locations in Fig. 1 are given because the other three values cannot be computed.

What ecological implications do the OOV transects (or gradients) have? We may compare the transects with Whittaker (1975) ecoclines described earlier. In particular, transect $\mathrm{L}_{8}$ (see Fig. 3 and Table 2) coincides with Whittaker's ecocline along an increasing aridity gradient (tropical rainforest $\rightarrow$ tropical seasonal forest $\rightarrow$ tropical broadleaf woodland $\rightarrow$ tropical $\quad$ scrub $\rightarrow$ warm semidesert $\rightarrow$ true desert), whereas transect $\mathrm{L}_{20}$ matches Whittaker's ecocline along an elevation gradient (tropical rainforest $\rightarrow$ temperate rainforest $\rightarrow$ elfinwood $\rightarrow$ alpine $\quad$ scrubland $\rightarrow$ alpine grassland). By shifting the reference point $(\mathrm{O})$ in Fig. 3 accordingly, the other ecoclines identified by Whittaker (1975) can also be recognized. However, the statistical thermodynamic model shows continuous, though nonlinear, vegetation changes in a unified thermodynamic state space (Figs. 1 and 2), while Whittaker's model simply places different biome types, as discrete entities, in a precipitation-temperature coordinate system. Furthermore, according to our statistical thermodynamic model, there are 31 distinctive vegetation gradients at the global scale, of which the empirically derived ecolines by Whittaker are only a subset.

\section{Discussion and conclusions}

Traditionally, empirical and inductive approaches have been dominant in the study of vegetation pattern at large scales. In this paper, we take a theoretical and deductive approach to develop a statistical thermodynamic model for quantifying the OOV and its large-scale patterns. 
The OOV may be understood as a macroscopic, thermodynamic representation of the steady state reached between vegetation and its environment over large spatial and temporal scales. In different geographical regions, groups of species form distinctive ecosystems or biomes as a result of the interactions between biotic communities and their physical environment as well as between the biological components themselves. Thus, world biomes have their characteristic values of $\mathrm{OOV}$ that can be determined primarily by mean annual AET and temperature (Eqs. (4) and (5)). In the thermodynamic state space, the values of OOV at all points form a continuous surface from which both within- and between-biome variations can be examined. The pattern of OOV corresponds to the pattern of world-biome types in relation to precipitation and temperature described by Whittaker (1975).

Our thermodynamic model of global vegetation pattern differs from Whittaker (1975) in several aspects. First of all, our approach is deductive and theoretical, whereas Whittaker's approach is inductive, empirical. In our model, the three-dimensional state space composed of $A$, AET and $T$ not only serves as a coordinate system that defines the boundaries of different vegetation types, but also unites these variables with the OOV in a thermodynamic framework. In essence, this is not a Newtonian space, but a 'field' whose properties

Table 2

Gradients of the OOV at the global scale

\begin{tabular}{|c|c|c|c|c|c|c|c|}
\hline Transect & Biomes on transect & Group & Type & Properties & $\mathrm{OOV}^{\prime} \eta=1.0$ & OOV' $^{\prime} \eta=0.8^{\prime}$ & OOV' $^{\prime} \eta=0.5$ \\
\hline $\mathrm{L}_{0}$ & 1 & 1 & 1 & $\mathrm{AET}=b T$ & 0.0000 & 0.0000 & 0.0000 \\
\hline $\mathrm{L}_{1}$ & 1 & 2 & 2 & $\mathrm{AET} \uparrow, T \uparrow, \mathrm{AET}<b T$ & -0.0152 & -0.0122 & -0.0076 \\
\hline $\mathrm{L}_{2}$ & 1 & & 3 & $\mathrm{AET}-, T \uparrow$ & -0.0281 & -0.0225 & -0.014 \\
\hline $\mathrm{L}_{3}$ & 1 & & 4 & $\mathrm{AET} \downarrow, T \uparrow$ & -0.4058 & -0.3246 & -0.2029 \\
\hline $\mathrm{L}_{4}$ & 1,2 & & & & -1.0018 & -0.8014 & -0.5009 \\
\hline $\mathrm{L}_{5}$ & $1,2,8$ & & & & -1.4404 & -1.1523 & -0.7202 \\
\hline $\mathrm{L}_{6}$ & $1,2,8,12,9$ & & & & -1.7246 & -1.3797 & -0.8623 \\
\hline $\mathrm{L}_{7}$ & $1,2,8,12,9,17,20$ & & & & -2.1506 & -1.7205 & -1.0753 \\
\hline $\mathrm{L}_{8}$ & $1,2,8,12,9,17,20$ & & 5 & $\mathrm{AET} \downarrow, T-$ & -2.1506 & -1.7205 & -1.0753 \\
\hline $\mathrm{L}_{9}$ & $1,2,8,12,9,17,20$ & & 6 & $\mathrm{AET} \downarrow, T \downarrow, \mathrm{AET}<b T$ & -2.1506 & -1.7205 & -1.0753 \\
\hline $\mathrm{L}_{10}$ & $1,2,8,12,9,18,20$ & & & & -2.1506 & -1.7205 & -1.0753 \\
\hline $\mathrm{L}_{11}$ & $1,2,8,12,11,18,20$ & & & & -2.1506 & -1.7205 & -1.0753 \\
\hline $\mathrm{L}_{12}$ & $1,2,10,13,11,18,20$ & & & & -2.1506 & -1.7205 & -1.0753 \\
\hline $\mathrm{L}_{13}$ & $1,2,4,5,10,13,11,18,20$ & & & & -2.1506 & -1.7205 & -1.0753 \\
\hline $\mathrm{L}_{14}$ & $1,4,5,10,13,11,18,20$ & & & & -2.1506 & -1.7205 & -1.0753 \\
\hline $\mathrm{L}_{15}$ & $1,3,4,5,10,13,11,18,20$ & & & & -2.1506 & -1.7205 & -1.0753 \\
\hline $\mathrm{L}_{16}$ & $1,3,4,5,6,10,13,11,18,20$ & & & & -2.1506 & -1.7205 & -1.0753 \\
\hline $\mathrm{L}_{17}$ & $1,3,4,5,6,10,13,11,19$ & & & & -2.1506 & -1.7205 & -1.0753 \\
\hline $\mathrm{L}_{18}$ & $1,3,4,5,6,10,13,15,19$ & & & & -2.1506 & -1.7205 & -1.0753 \\
\hline $\mathrm{L}_{19}$ & $1,3,6,16,15,19$ & & & & -2.1506 & -1.7205 & -1.0753 \\
\hline $\mathrm{L}_{20}$ & $1,3,7,22,14,21$ & & & & -2.1506 & -1.7205 & -1.0753 \\
\hline $\mathrm{L}_{21}$ & $1,3,7,22,14$ & & & & -1.5768 & -1.2614 & -0.7884 \\
\hline $\mathrm{L}_{22}$ & $1,3,7,22$ & & & & -1.353 & -1.0824 & -0.6765 \\
\hline $\mathrm{L}_{23}$ & $1,3,7$ & & & & -1.1881 & -0.9505 & -0.594 \\
\hline $\mathrm{L}_{24}$ & 1,3 & & & & -0.4732 & -0.3786 & -0.2366 \\
\hline $\mathrm{L}_{25}$ & 1 & & & & -0.3413 & -0.273 & -0.1706 \\
\hline $\mathrm{L}_{26}$ & 1 & 3 & 7 & $\mathrm{AET} \downarrow, T \downarrow, \mathrm{AET}>b T$ & 0.014 & 0.0112 & 0.007 \\
\hline $\mathrm{L}_{27}$ & 1 & & 8 & $\mathrm{AET}-, T \downarrow$ & 0.0272 & 0.0218 & 0.0136 \\
\hline $\mathrm{L}_{28}$ & 1 & & 9 & $\mathrm{AET} \uparrow, T \downarrow$ & 0.1612 & 0.129 & 0.0806 \\
\hline $\mathrm{L}_{29}$ & 1 & & 10 & $\mathrm{AET} \uparrow, T-$ & 0.4326 & 0.3461 & 0.2163 \\
\hline $\mathrm{L}_{30}$ & 1 & & 11 & $\mathrm{AET} \uparrow, T \uparrow, \mathrm{AET}>b T$ & 0.273 & 0.2184 & 0.1365 \\
\hline
\end{tabular}

The actual values of OOV' are those listed in the table times $10^{33}$. The names of the world biomes are given in Fig. 1. Thirty-one vegetation gradients in terms of OOV are derived (also see Fig. 3). 
are influenced by the matter within. In the threedimensional thermodynamic state space, each point corresponds to a particular value of the OOV, and within-biome changes can also be detected by the variations of OOV values. Furthermore, because all biomes are now classified according to their OOV values, they are directly comparable. Also, because empirically observed ecoclines can only be a subset of all possible gradients of ecosystems at a particular scale (e.g. world-wide biomes), the statistical thermodynamic model provides a theoretical means to derive the maximum possible ecoclines. In general, as we deal with the complexity, diversity, and heterogeneity of ecological systems, statistical and non-equilibrium thermodynamics may serve as both a theoretical framework and a practical modeling approach to integrate patterns and processes across scales.

\section{Acknowledgements}

We would like to thank Felix Müller, Canran Liu, and Bailian Li for their valuable comments on an earlier version of the paper. This research was supported by grants from Chinese Natural Science Foundation (Project 39560023 to H.Z. and Project 30028002 to J.W.). J.W.'s research was also in part supported through a grant from US Environmental Protection Agency grant R827676-01-0. This paper has not been subjected to the Agencies' required peer and policy review and therefore does not necessarily reflect the views of the agencies and no official endorsement should be inferred.

\section{References}

Atmar, W., 2001. A profoundly repeated pattern. Bulletin of Ecological Society of America 82, 208-211.

Barbour, M.G., Billings, W.D. (Eds.), 2000. North American Terrestrial Vegetation. Cambridge University Press, New York, p. 708.

Callen, H.B., 1985. Thermodynamics and An Introduction to Thermostatistics. Wiley, New York, p. 493.

Crawly, M.J., 1997. Plant Ecology. Blackwell, Oxford, p. 717.
Currie, D.J., Paquin, V., 1987. Large-scale biogeographical patterns of species richness of trees. Nature 329, 326-327.

Currie, D.J., 1991. Energy and large-scale patterns of animaland plant-species richness. American Naturalist 137, $27-$ 49.

Durrett, R., Levin, S.A., 1994a. Stochastic spatial models: a user's guide to ecological applications. Philosophical Transactions of the Royal Society of London 343 (Series B), 329-350.

Durrett, R., Levin, S.A., 1994b. The importance of being discrete (and spatial). Theoretical Population Biology 46, $363-394$.

Fränzle, O., 1994. Thermodynamic aspects of species diversity in tropical and ectropical plant communities. Ecological Modelling 75/76, 63-70.

Hill, T.L., 1960. An Introduction to Statistical Thermodynamics. Addison-Wesley, Reading, p. 508.

Holdridge, L.R., 1947. Determination of world plant formations from simple climatic data. Science 105, 367-368.

Jorgensen, S.E., Patten, B.C., Straskraba, M., 1992. Ecosystems emerging: toward an ecology of complex systems in a complex future. Ecological Modelling 62, 1-27.

Jorgensen, S.E., 1997. Integration of Ecosystem Theories: A Pattern. Kluwer Academic Publishers, Boston, p. 388.

Levin, S.A., 1999. Fragile Dominion: Complexity and the Commons. Perseus Books, Reading, p. 250.

Li, B.-L., 2000. Why is the holistic approach becoming so important in landscape ecology? Landscape and Urban Planning 50, 27-41.

May, R.M., 1986. The search for patterns in the balance of nature: advances and retreats. Ecology 67, 1115-1126.

Müller, F., 1997. State-of-the-art in ecosystem theory. Ecological Modelling 100, 135-161.

Naveh, Z., Lieberman, A.S., 1984. Landscape Ecology: Theory and Application. Springer, New York, p. 356.

Naveh, Z., 1987. Biocybernetic and thermodynamic perspectives of landscape functions and land use patterns. Landscape Ecology 1, 75-83.

Nicolis, G., Prigogine, I., 1977. Self-Organization in Nonequilibrium Systems: From Dissipative Structures to Order through Fluctuations. Wiley, New York, p. 491.

Nicolis, G., Prigogine, I., 1989. Exploring Complexity: An Introduction. W.H. Freeman and Company, New York, p. 313.

O’Neill, R.V., DeAngelis, D.L., Waide, J.B., Allen, T.F.H., 1986. A Hierarchical Concept of Ecosystems. Princeton University Press, Princeton, p. 253.

Pickett, S.T.A., Collins, S.L., Armesto, J.J., 1987. A hierarchical consideration of causes and mechanisms of succession. Vegetatio 69, 109-114.

Rosenzweig, M.L., 1968. Net primary productivity of terrestrial communities: Prediction from climatological data. The American Naturalist 102, 67-74.

Schneider, E., Kay, J.J., 1994. Complexity and thermodynamics: Towards a new ecology. Futures 24, 626-647. 
Schrödinger, E., 1944. What Is Life? Cambridge University Press, London.

Seller, W.D., 1965. Physical Climatology. University of Chicago Press, Chicago, 272 pp.

Ulanowicz, R.E., 1997. Ecology, The Ascendent Perspective. Columbia University Press, New York, p. 201.

Volterra, V., 1926. Fluctuations in the abundance of a species considered mathematically. Nature 112, 118.

Whittaker, R.H., 1975. Communities and Ecosystems. Macmillan, New York, p. 385.

Wu, J., 1991. Dissipative structure, hierarchy theory and ecosystems. Chinese Journal of Applied Ecology 2, 181186.

Wu, J., Levin, S.A., 1994. A spatial patch dynamic modeling approach to pattern and process in an annual grassland. Ecological Monographs 64 (4), 447-464.

Wu, J., Loucks, O.L., 1995. From balance-of-nature to hierarchical patch dynamics: A paradigm shift in ecology. Quarterly Review of Biology 70, 439-466.

Wu, J., 1999. Hierarchy and scaling: Extrapolating information along a scaling ladder. Canadian Journal of Remote Sensing 25, 367-380. 